\title{
Lagrangian chaotic saddles and objective vortices in solar plasmas
}

\author{
Abraham C.-L. Chian $\odot,{ }^{1,2,3, *}$ Suzana S. A. Silva $\odot,{ }^{4}$ Erico L. Rempel $\odot,{ }^{3,4}$ Luis R. Bellot Rubio $\odot,{ }^{5}$ Milan Gošić $\odot,{ }^{6,7}$ \\ Kanya Kusano $\mathbb{0}^{1}{ }^{1}$ and Sung-Hong Park ${ }^{1}{ }^{1}$ \\ ${ }^{1}$ Institute for Space-Earth Environmental Research (ISEE), Nagoya University, Furo-cho, Chikusa-ku, Nagoya 464-8601, Japan \\ ${ }^{2}$ School of Mathematical Sciences, University of Adelaide, Adelaide, SA 5005, Australia \\ ${ }^{3}$ National Institute for Space Research (INPE), P.O. Box 515, São José dos Campos, São Paulo 12227-010, Brazil \\ ${ }^{4}$ Institute of Aeronautical Technology (ITA), São José dos Campos, São Paulo 12228-900, Brazil \\ ${ }^{5}$ Instituto de Astrofísica de Andalucía (CSIC), Apartado de Correos 3004, E-18080 Granada, Spain \\ ${ }^{6}$ Lockheed Martin Solar and Astrophysics Laboratory, Palo Alto, California 94304, USA \\ ${ }^{7}$ Bay Area Environmental Research Institute, Moffett Field, California 94035, USA
}

(Received 11 September 2020; accepted 18 October 2020; published 3 December 2020)

\begin{abstract}
We report observational evidence of Lagrangian chaotic saddles in plasmas, given by the intersections of finite-time unstable and stable manifolds, using an $\approx 22 \mathrm{~h}$ sequence of spacecraft images of the horizontal velocity field of solar photosphere. A set of 29 persistent objective vortices with lifetimes varying from 28.5 to 298.3 min are detected by computing the Lagrangian averaged vorticity deviation. The unstable manifold of the Lagrangian chaotic saddles computed for $\approx 11 \mathrm{~h}$ exhibits twisted folding motions indicative of recurring vortices in a magnetic mixed-polarity region. We show that the persistent objective vortices are formed in the gap regions of Lagrangian chaotic saddles at supergranular junctions.
\end{abstract}

DOI: 10.1103/PhysRevE.102.060201

Recent advances in dynamical systems and chaotic advection allow in-depth analyses of the formation and evolution of vortices in turbulent flows in fluids and plasmas. In stationary vector fields, unstable and stable manifolds form transport barriers that are invariant under the flow, being responsible for stretching and folding in the mixing of passive tracers. In time-varying vector fields, the corresponding version of unstable and stable manifolds of particle trajectories constitute distinguished material lines or surfaces that act as transport barriers that are invariant in the extended phase space of position and time. These distinguished lines or surfaces are the hyperbolic Lagrangian coherent structures that attract or repel the neighboring material [1], both retarding and facilitating transport fluxes. Hence, they are responsible for organizing and mediating the transport and interaction of matter and energy in the flow. In particular, the attracting and repelling hyperbolic Lagrangian coherent structures act as transport barriers that enable the formation of the elliptic Lagrangian coherent structures (vortices) [2-4]. Lagrangian coherent structures have been extensively studied in atmosphere and ocean [1,5] and have been investigated by numerical simulation of nuclear fusion [6] and ionospheric [7] and astrophysical [3,8,9] plasmas, as well as observation of laboratory [10], ionospheric [11], and solar [4,12-16] plasmas.

Chaotic saddles given by the intersections of unstable and stable manifolds are chaotic invariant sets in the phase space that are not attracting, which are related to transient chaos and fractal patterns in the advection dynamics [17] as well

\footnotetext{
*abraham.chian@gmail.com
}

as edge of chaos [18]. The phenomenon of chaotic saddles in plasmas has been studied in the theory of tokamaks [19], Alfvén waves [20], drift waves [21], magnetorotational instability [22], and magnetoconvection [23]. Lagrangian chaotic saddles have been observed in atmospheric and laboratory studies of chaotic advection [24].

Explosive and eruptive events with multiscale spatiotemporal dynamics such as flares and coronal mass ejections in the active Sun [25] and microflares and minicoronal mass ejections in the quiet Sun [26] are driven by photospheric vortical flows in the vicinity of the polarity inversion lines and at supergranular junctions, respectively. Hence, the study of the origin and evolution of long-lived vortices in the solar turbulence is fundamental for understanding physical processes that may lead to coronal eruptions. Solar supergranulations can originate from thermal magnetoconvection processes such as magnetized Rayleigh-Bénard convection [14]. Chaotic saddles have been studied in numerical simulations of Rayleigh-Bénard convection [27] and in a reduced model of magnetoconvection [23].

The aim of this paper is twofold: (1) to investigate the genesis and life cycle of persistent objective vortices at supergranular junctions in the quiet Sun using Hinode spacecraft imaging of the photosphere and (2) to report the observation of Lagrangian chaotic saddles in solar plasmas and elucidate their pivotal role in the nonlinear dynamics of persistent vortices in supergranular turbulence.

The analysis is done using the photospheric horizontal velocity fields deduced by the local correlation tracking method from an $\approx 22 \mathrm{~h}$ sequence of Hinode continuum intensity images of the Sun's disk center, with a cadence of $90 \mathrm{~s}$, from 08:31:15 UT on 2010 November 2 to 06:19:42 UT on 2010 
November $3[4,15,28,29]$. Despite the limitation of the local correlation tracking method in underestimating the velocity fields, it provides a useful proxy for reconstructing many morphological features of the solar plasma flow [30]. In a previous paper [4], Lagrangian coherent structures in the photosphere were studied for an $\approx 7 \mathrm{~h}$ reduced data set, which is not long enough to identify Lagrangian chaotic saddles with precision. In this paper, we are able to detect Lagrangian chaotic saddles using a long data set of $\approx 22 \mathrm{~h}$. In particular, the unstable manifold of the Lagrangian chaotic saddles computed for $\approx 11 \mathrm{~h}$ in this paper is capable of demonstrating the twisted folding motions associated with the recurrence of persistent vortices in a magnetic mixed-polarity region which is not possible with the $\approx 7 \mathrm{~h}$ reduced dataset. Moreover, in the previous paper [4] the elliptic Lagrangian coherent structures were detected using prefixed durations of 15 and $60 \mathrm{~min}$ without obtaining any information about the vortex life cycle. In this paper, we carry out a comprehensive survey of long-duration elliptic Lagrangian coherent structures in the region of a supergranulation and its neighborhood by determining systematically the initial and final times of a set of 29 persistent objective vortices and measuring their properties during their lifecycles.

First, we detect the hyperbolic Lagrangian coherent structures in supergranular turbulence by computing the backward and forward finite-time Lyapunov exponent (b-FTLE/f-FTLE) [31] for an interval of $\approx 11 \mathrm{~h}$, respectively, of the horizontal velocity field and advecting a dense grid of $405 \times 405$ tracer particles over the domain of interest. Note that the finite-time Lyapunov exponent is objective, i.e., independent of the observer's frame of reference [1]. Advecting a particle backward in time reveals the attracting hyperbolic Lagrangian coherent structures (time-dependent unstable manifolds) in the b-FTLE field, whereas advecting a particle forward in time reveals the repelling hyperbolic Lagrangian coherent structures (timedependent stable manifolds) in the f-FTLE field. Figure 1(a) shows the plot of b-FTLE using a sequence of 436 frames of the horizontal velocity field, calculated backward in time from 19:25:29 UT to 08:31:15 UT on 2 November 2010, which unravels the time-dependent Lagrangian boundaries of supergranular cells. Thin ridges of large positive b-FTLE in Fig. 1(a) represent the locally strongest attracting material lines that exert the most influence on the converging transport of supergranular flows in the given time interval. These ridges act as sinks for the down draft of photospheric flows that lead to vortical motions. Chian et al. $[4,13]$ demonstrated the link of the attracting hyperbolic Lagrangian coherent structures to the network of intense concentration of magnetic fluxes at supergranular junctions. Figure 1(a) shows that the area of observation covers a section of solar surface containing one supergranular cell and its surroundings.

Figure 1(b) shows the plot of f-FTLE computed forward in time from 19:25:29 UT on 2010 November 2 to 06:19:42 UT on 2010 November 3, using a sequence of 436 frames of the horizontal velocity field, to yield the repelling hyperbolic Lagrangian coherent structures. The black pluses denote the locations of time-dependent Lagrangian centers of supergranular cells determined by the local maxima of f-FTLE [4]. Thin ridges of large positive f-FTLE in Fig. 1(b) represent the locally strongest repelling material lines which exert the most
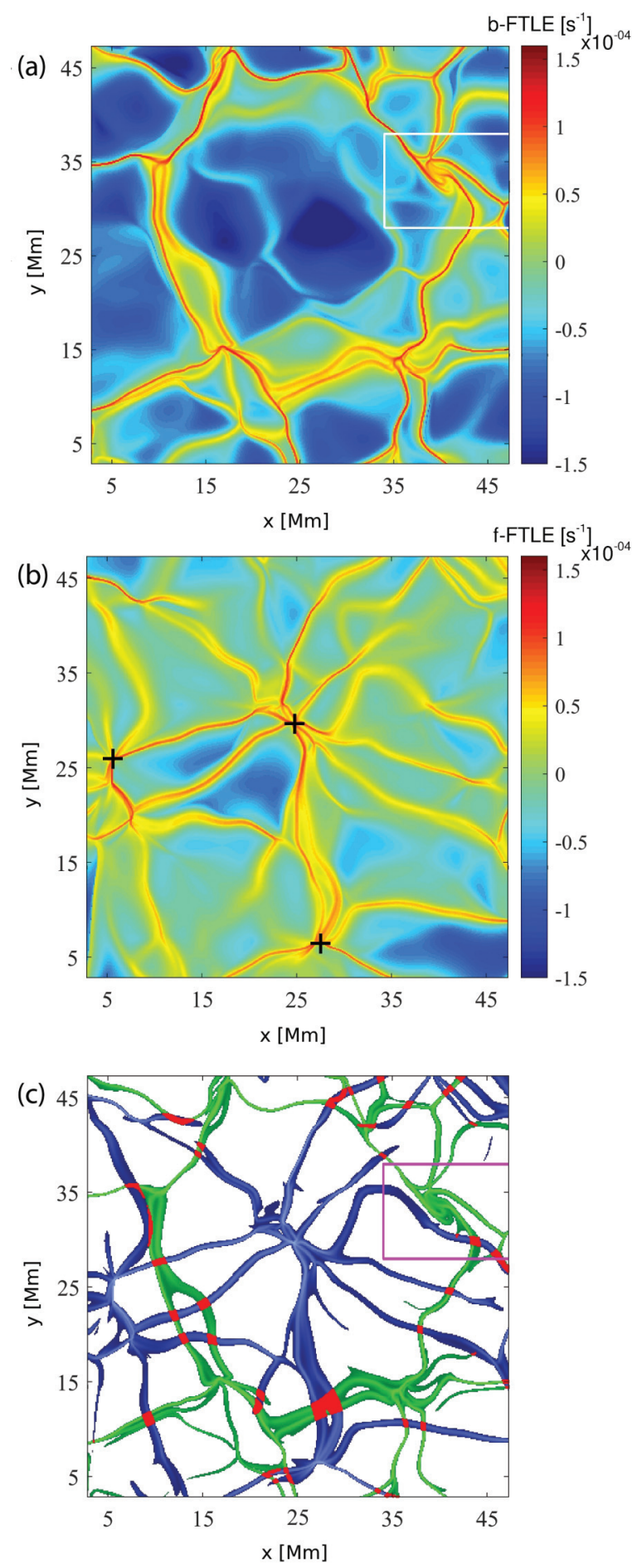

FIG. 1. Lagrangian chaotic saddles. (a) Finite-time unstable manifolds given by b-FTLE computed from 19:25:29 UT on 2010 November 2 to $08: 31: 15$ UT on 2010 November 2. (b) Finite-time stable manifolds given by f-FTLE computed from 19:25:29 UT on 2010 November 2 to 06:19:42 UT on 2010 November 3. The black pluses mark the Lagrangian centers of supergranular cells. (c) Lagrangian chaotic saddles (red points) determined by the intersections of the thresholded finite-time unstable (green) and stable (blue) manifolds obtained from Figs. 1(a) and 1(b), respectively, with the threshold of b-FTLE (f-FTLE) given by $0.3 \times 10^{-4}\left(0.25 \times 10^{-4}\right)$, respectively. The white/magenta box marks a mixed-polarity region of magnetic fields dominated by vortical flows. 
influential impact on the diverging transport of supergranular flows in the given time interval. These ridges provide the transport barriers that facilitate the formation of vortices and concentration of strong magnetic fields in the valleys (i.e., low-value regions) of f-FTLE. Yeates et al. [12] and Chian et al. [13] demonstrated the link of f-FTLE to the squashing factor, which identifies the most likely sites for the occurrence of magnetic reconnection [32].

In Fig. 1(c), we superpose the thresholded unstable (green) and stable (blue) manifolds given by Figs. 1(a) and 1(b), respectively. Lagrangian chaotic saddles, denoted by the set of red points, are given by the intersections of finite-time unstable and stable manifolds. The void region of Lagrangian chaotic saddles along the unstable manifolds in Fig. 1(c) where the red points are absent is known as the gap region. It follows from Fig. 1 that the stable manifolds connecting the Lagrangian centers of supergranular cells [4] contribute to the formation of Lagrangian chaotic saddles, which play a key role in the Lagrangian skeleton of turbulence [33] in the photosphere.

The computation of the backward and forward finite-time Lyapunov exponents for a long duration of $\approx 11 \mathrm{~h}$ yields sharp images of the ridges of the hyperbolic Lagrangian coherent structures seen in Figs. 1(a) and 1(b). The thresholded plots of Fig. 1(c) facilitate the visualization of Lagrangian chaotic saddles and the fine details of Lagrangian coherent structures. Note that in reality a chaotic saddle contains an infinite number of points, whereas only about 30 points are seen in Fig. 1(c). This is due to the finite resolution and duration of the solar data set available for computing the manifolds in this study and the threshold values chosen for their plots. In the future, efforts should be made to find other examples of Lagrangian chaotic saddles with a larger number of points in observation and numerical simulation of plasma turbulence. It is pertinent to mention that the intersections between the unstable and stable manifolds illustrated in Fig. 1(c) cannot be directly related to the points of a chaotic saddle in stationary or periodic flows. The repelling Lagrangian coherent structures (LCS) are obtained from an FTLE field computed from $t_{0}$ to $t_{0}+\tau$, whereas the attracting LCS are computed from $t_{0}$ to $t_{0}-\tau$. Both reflect LCS at $t_{0}$, but since our flow is not periodic, the dynamics in the forward and backward time intervals are quite different and the hyperbolic LCS computed over $\left[t_{0}-\tau, t_{0}\right]$ and $\left[t_{0}, t_{0}+\tau\right]$ do not evolve into each other as $t_{0}$ is varied. An alternative approach is to extract the repelling LCS at the initial time $t_{0}-\tau$ and extract the attracting LCS at $t_{0}+\tau$, then obtain the repelling LCS at $t_{0}$ by advecting the repelling LCS from $t_{0}-\tau$ to $t_{0}$ under the flow and obtain the attracting LCS at $t_{0}$ by advecting the attracting LCS from $t_{0}+\tau$ to $t_{0}$ under the flow. However, as mentioned by Farazmand and Haller [34], this second approach has serious numerical issues. A third approach mentioned by Jánosi and Tél [35] to obtain manifolds in unsteady flows is not based on the FTLE fields but on the integration of initial conditions in special directions in the flow. The unstable manifolds are obtained by integrating a set of initial conditions on a short line segment along an eigenvector of a hyperbolic point forward in time from $t_{0}-\tau$ to $t_{0}$ and the stable manifolds by integrating another set backward in time from $t_{0}+\tau$ to $t_{0}$. In this way, the crossings between manifolds belong to the same time instant $t_{0}$. The difficulty here is to accurately find the appropriate hyperbolic points and their eigenvectors from an observational field.

We detect objective vortices by computing the elliptic Lagrangian coherent structures with the aid of Eulerian instantaneous vorticity deviation (IVD) [2,3] of a particle,

$$
\operatorname{IVD}(\mathbf{x}, t)=|\omega(\mathbf{x}, t)-\bar{\omega}(t)|,
$$

where the vorticity $\omega=\nabla \times \mathbf{u}$ and $\bar{\omega}(t)$ is the instantaneous spatial mean of $\omega$, and the Lagrangian averaged vorticity deviation (LAVD) $[2-4,15]$ as the integrated IVD along a fluid particle trajectory at the position $\mathbf{x}_{0}$ from an initial time $t_{0}$ to a final time $t_{0}+\tau$,

$$
\operatorname{LAVD}_{t_{0}}^{t_{0}+\tau}\left(\mathbf{x}_{0}\right)=\int_{t_{0}}^{t_{0}+\tau}|\omega(\mathbf{x}(s), s)-\bar{\omega}(s)| d s .
$$

Haller et al. [1] proved that the Eulerian instantaneous vorticity deviation and the Lagrangian averaged vorticity deviation are objective. A set of laboratory experiments performed with magnetic-stirrer-generated vortices have provided evidence in support of the theory of vortices based on the LAVD [36]. We use the d-criterion introduced by Silva et al. [15] to avoid false vortex detection. The three-dimensional (3D) simulations of solar vortex tubes generated by convective flows and shear flows driven by Kelvin-Helmholtz instability [37] show that both vertical and horizontal vortex tubes are generated, but the vertical vortex tubes predominate as the horizontal vortex tubes are often dragged into the down-flow regions. Hence, our use of purely horizontal velocity fields in the photosphere derived from the spacecraft observations, which yield purely vertical vorticities, is justified.

In Fig. 2(a), we show an example of an Eulerian objective vortex by computing the Eulerian instantaneous vorticity deviation for the initial time of a vortex (M1) at 17:23:57 UT on 2010 November 2 in the same white/magenta box region of Fig. 1, where the center (boundary) of the Eulerian objective vortex is indicated by the magenta cross (line), respectively. In Fig. 2(b), we show an example of a Lagrangian objective vortex computed by the Lagrangian averaged vorticity deviation for the lifetime of a vortex (M1) from 17:23:57 UT to 22:22:32 UT on 2010 November 2 in the same white/magenta box region of Fig. 1, where the center (boundary) of the Lagrangian vortex is indicated by the magenta cross (line), respectively. Note that in contrast to the Eulerian vortex detected by the Eulerian instantaneous vorticity deviation method in Fig. 2(a), which is a snapshot at the initial vortex time, the Lagrangian vortex detected by the Lagrangian averaged vorticity deviation method in Fig. 2(b) is time dependent and averaged over the vortex lifetime of $\approx 5 \mathrm{~h}$.

Next, we apply the Lagrangian averaged vorticity deviation technique to perform a systematic investigation of the genesis of persistent objective vortices for the $\approx 22 \mathrm{~h}$ Hinode data, from 08:31:15 UT on 2010 November 2 to 06:19:42 UT on 2010 November 3. First, we identify the initial and final times for the lifetime of each persistent objective vortex. Then, we determine the boundary and center of each vortex by computing the LAVD from its initial time to final time. Figure 2(c) shows the set of 29 persistent objective vortices detected for the entire duration, with lifetimes ranging from $28.5 \mathrm{~min}$ (vortex R14) to $298.3 \mathrm{~min}$ (vortex M1), superposed 
(a)

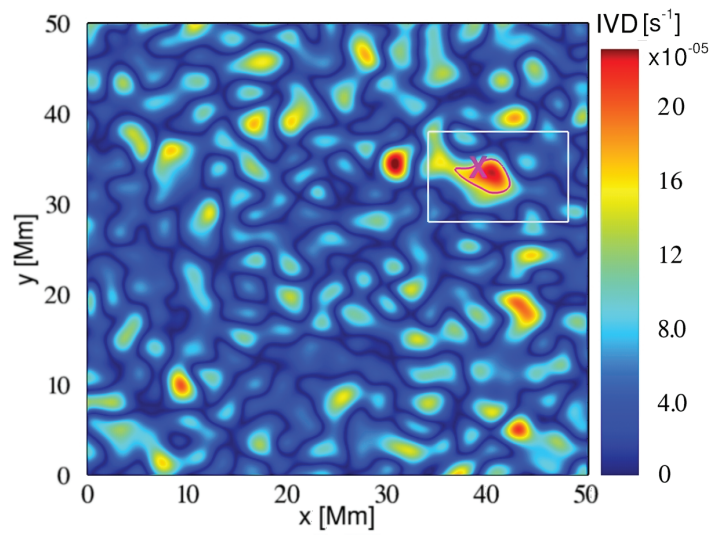

(b)

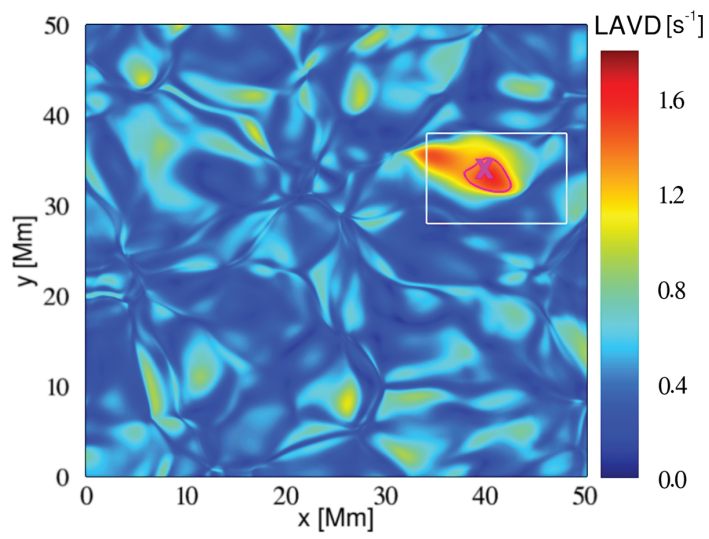

(c)

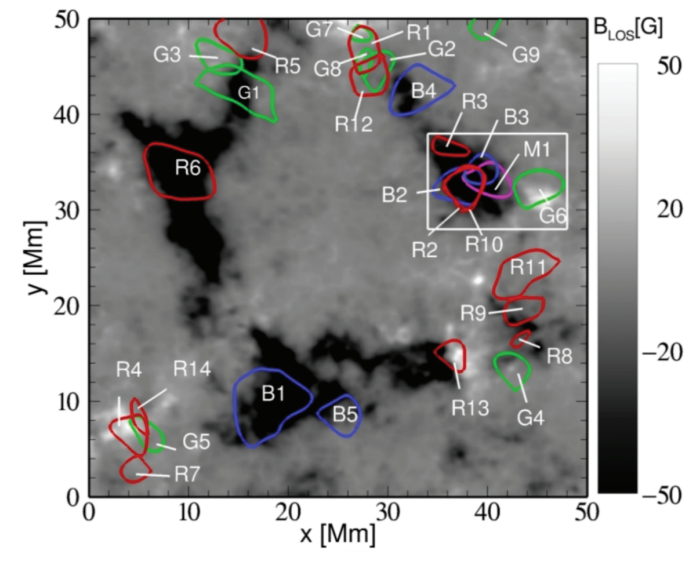

FIG. 2. Objective vortices. (a) IVD computed at the initial time of vortex M1 at 17:23:57 UT on 2010 November 2, where the vortex center (boundary) is indicated by the magenta cross (line), respectively. (b) LAVD computed for the lifetime of vortex M1 from 17:23:57 UT to 22:22:32 UT on 2010 November 2, where the vortex center (boundary) is indicated by the magenta cross (line), respectively. (c) The boundary of 29 persistent objective vortices detected by LAVD from 08:31:15 UT on 2010 November 2 to 06:19:42 UT on 2010 November 3 , superposed by the mean line-of-sight magnetic field for the same time interval consisted of 1 vortex with lifetime $(T) \approx 5 \mathrm{~h}$ (magenta, M), 5 vortices with $3.5 \mathrm{~h}>T>1.6 \mathrm{~h}$ (blue, B), 9 vortices with $1.6 \mathrm{~h}>T>0.9 \mathrm{~h}$ (green, G), and 14 vortices with $0.9 \mathrm{~h}>T>0.47 \mathrm{~h}$ (red, R). The white box marks the same magnetic mixed-polarity region as Fig. 1 where a sequence of 7 recurrent persistent objective vortices are detected during the entire time interval.
TABLE I. A sequence of seven recurrent persistent objective vortices detected from 09:11:46 UT on 2010 November 2 to 05:10:39 UT on 2010 November 3 in a magnetic mixed-polarity region of supergranular junctions.

\begin{tabular}{lcccccc}
\hline \hline & $t_{0}$ & $t_{f}$ & $T(\mathrm{~min})$ & I-IVD & P-LAVD & T-LAVD \\
\hline R2 & $09: 11: 46$ & $10: 02: 47$ & 51 & $9.15 \times 10^{-5}$ & 0.30 & 169.57 \\
R10 & $10: 16: 17$ & $10: 52: 18$ & 36 & $1.18 \times 10^{-4}$ & 0.23 & 139.31 \\
B2 & $11: 23: 49$ & $14: 23: 53$ & 180 & $8.67 \times 10^{-5}$ & 1.08 & 942.46 \\
M1 & $17: 23: 57$ & $22: 22: 32$ & 298.3 & $1.93 \times 10^{-1}$ & 1.57 & 1230 \\
G6 & $23: 43: 33$ & $00: 43: 55$ & 60 & $1.06 \times 10^{-4}$ & 0.39 & 299.32 \\
R3 & $00: 09: 03$ & $00: 58: 35$ & 49.5 & $8.16 \times 10^{-5}$ & 0.23 & 70.45 \\
B3 & $02: 48: 07$ & $05: 10: 39$ & 142.5 & $8.25 \times 10^{-5}$ & 0.80 & 412.23 \\
\hline \hline
\end{tabular}

by the mean line-of-sight magnetic field for the whole time interval. Table I presents an overview of life-cycle properties of a sequence of seven recurrent persistent objective vortices detected in a mixed-polarity region enclosed by the white box in Fig. 2(c). We list the vortices in the chronological order of their respective initial time $\left(t_{0}\right)$ and also include their final time $\left(t_{f}\right)$, lifetime $(T)$, initial value of the Eulerian instantaneous vorticity deviation (I-IVD), local maximum value of the Lagrangian averaged vorticity deviation (P-LAVD), and the Lagrangian averaged vorticity deviation integrated over the lifetime (T-LAVD). This information can be useful for probing the formation and evolution of vortices; e.g., it shows that the vortex M1 that has the longest lifetime among the sequence of seven vortices has the largest values of I-IVD, P-LAVD, and T-LAVD.

In Figs. 3(a), 3(b), respectively, we superpose the unstable (stable) manifolds given by Figs. 1(a), 1(b) with the boundaries (magenta line) of the persistent objective vortices extracted from Fig. 2(c) whose lifetimes fall into the time interval of $\approx 11 \mathrm{~h}$ of the respective invariant manifolds. In Figs. 3(c), 3(d), respectively, we superpose the thresholded unstable (stable) manifolds with the centers (magenta cross) of the Lagrangian vortices extracted from Fig. 2(c) whose lifetimes fall into the time interval of $\approx 11 \mathrm{~h}$ of the respective invariant manifolds. The background of Figs. 3(c), 3(d) displays the line-of-sight magnetic field time averaged over the respective time interval of the unstable (stable) manifolds. In Figs. 3(a) and 3(c), the total number of persistent objective vortices is 16, whereas in Figs. 3(b) and 3(d), the total number of persistent objective vortices is 13. It follows from Figs. 3(a) and 3(c) that the persistent objective vortices are mostly located in the high-value regions of b-FTLE, where the attracting hyperbolic Lagrangian coherent structures provide the sinks for photospheric flows.

Analysis of Figs. 3(a) and 3(c) shows that the persistent objective vortices and intense concentrations of magnetic fluxes are located in the gap regions of the supergranular Lagrangian chaotic saddle along its unstable manifold. Although a few vortices are seen along the stable manifold of Figs. 3(b) and $3(d)$, it has been shown that all supergranular persistent vortices are located at the ridges of the unstable manifold (see Fig. 6 in Ref. [4]). Moreover, it is seen in Fig. 3(d) that the 

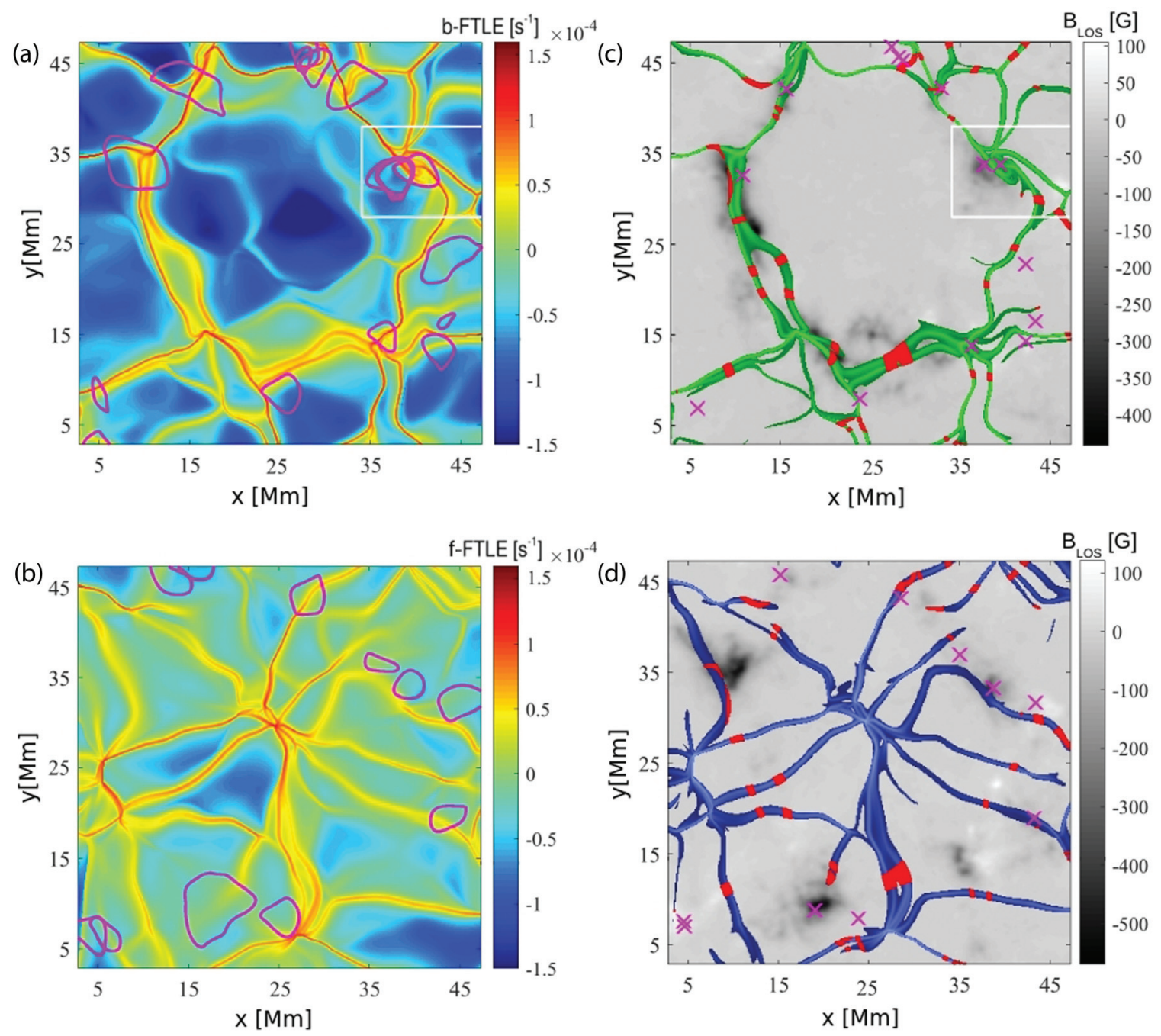

FIG. 3. Lagrangian view of photospheric turbulence at disk center of the quiet Sun. (a) The vortex boundary (magenta line) of persistent objective vortices detected from 08:31:15 UT to 19:25:29 UT on 2010 November 2, superposed by the finite-time unstable manifolds of Fig. 1(a). (b) The vortex boundary of persistent objective vortices detected from 19:25:29 UT on 2010 November 2 to 06:19:42 UT on 2010 November 3, superposed by the finite-time stable manifolds of Fig. 1(b). The vortex center (magenta cross) of persistent objective vortices, Lagrangian chaotic saddles (red), and the background line-of-sight magnetic field averaged over the time interval of the respective unstable and stable manifolds, superposed by (c) the thresholded finite-time unstable manifolds (green) with a threshold of $0.3 \times 10^{-4}$ and (d) the thresholded finite-time stable manifolds (blue) with a threshold of $0.25 \times 10^{-4}$. The white box marks the same magnetic mixed-polarity region as in Figs. 1 and 2.

vortex center of these vortices is away from the Lagrangian chaotic saddles. Hence, these vortices are also located in the gap regions. In the white box region of Figs. 1(a), 3(a), and 3(c) and the magenta box region of Fig. 1(c), we see that the unstable manifolds exhibit twisted folding motions resulting from the velocity shear driven by the converging down drafts from various nearby supergranular cells. These folded unstable manifolds attract plasma particles to the spiral regions of photospheric flow, giving rise to the recurrence of vortices seen in the magnetic mixed-polarity region of Fig. 3(c). The stable manifolds transport particles in the direction of the hyperbolic points of a chaotic saddle, where the particles undergo a transient chaotic motion before being transported to the direction of the unstable manifolds. This mixing process mediated by the Lagrangian chaotic saddles is the key to the genesis of persistent objective vortices as elucidated by numerical plasma simulations, which show that an elliptic Lagrangian coherent structure (vortex) is confined by the transport barriers consisted of attracting and repelling hyperbolic Lagrangian coherent structures (see Figs. 1 and 4 in Rempel et al. [3]). The visualization of the folded structures of the unstable manifolds in Figs. 1(a), 3(a), and 3(c) are possible thanks to the long duration computation of $\approx 11 \mathrm{~h}$ carried out in this paper. In contrast, this important feature of vortical motions is missing in the unstable manifolds computed for a shorter duration of $\approx 7 \mathrm{~h}$ (see Fig. 4(b) of Ref. [4]).

In conclusion, we presented observational evidence of Lagrangian chaotic saddles in plasmas, which confirms the previous theoretical predictions that chaotic saddles play a significant role in the nonlinear dynamics of stellar magnetoconvection [23,27] and in the genesis of turbulence in fluids 
and plasmas $[17,18]$. Our dynamical systems approach paves the way for further detection of Lagrangian coherent structures and Lagrangian chaotic saddles in laboratory, space, and astrophysical plasmas, as well as in fluids in general. This paper only considered 2D velocity fields due to the restriction of spacecraft observations. Future studies of Lagrangian chaotic saddles can be extended to $3 \mathrm{D}$ velocity and magnetic fields in numerical simulations and experimental observations of plasmas. Vortices are associated with a wealth of dynamical phenomena at supergranular junctions in the quiet Sun such as bright points, jets, minifilaments, microflares, and minicoronal mass ejections [26]. Our nonlinear dynamics methodology can be readily applied to study the formation and development of persistent vortices in the vicinity of the polarity inversion lines in active regions to improve the monitoring and forecasting of solar flares and coronal mass ejections [25,38,39], as well as to study atmospheric and oceanic cyclogenesis, thus providing a powerful tool for the prediction of space weather [40], weather [41], and ocean dynamics [42].

We thank the Hinode Mission for providing the data and the referee for valuable comments. This work was supported by Coordenação de Aperfeiçoamento de Pessoal de Nível Superior (CAPES No. 88882.316962/2019-01 and No. 88881.309066/2018-01, Brazil), Conselho Nacional de Desenvolvimento Científico e Tecnológico (CNPq, Brazil), NASA Contract No. NNM07AA01C [Solar-B (Hinode) Focal Plane Package Phase E], Ministério de Ciência, Innovación y Universidades (No. RTI2018-096886-B-C51, Spain), European Regional Development Fund (FEDER), and Center of Excellence Severo Ochoa Award to the Instituto de Astrofísica de Andalucía (No. SEV-2017-0709, Spain).
[1] G. Haller, Annu. Rev. Fluid Mech. 47, 137 (2015).

[2] G. Haller, A. Hadjighasem, M. Farazmand, and F. Huhn, J. Fluid Mech. 795, 136 (2016).

[3] E. L. Rempel, A. C.-L. Chian, F. J. Beron-Vera, S. Szanyi, and G. Haller, MNRAS 466, L108 (2017).

[4] A. C.-L. Chian, S. S. A. Silva, E. L. Rempel, M. Gošić, L. R. Bellot Rubio, K. Kusano, R. A. Miranda, and I. S. Requerey, MNRAS 488, 3076 (2019).

[5] S. Wiggins, Annu. Rev. Fluid Mech. 37, 295 (2005).

[6] K. Padberg, T. Hauff, F. Jenko, and O. Junge, New J. Phys. 9, 400 (2007); F. Pegoraro, D. Bonfiglio, S. Cappello, G. Di Giannatale, M. V. Falessi, D. Grasso, and M. Veranda, Plasma Phys. Control. Fusion 61, 044003 (2019).

[7] W. Tang and A. Mahalov, Phys. Plasmas 20, 032305 (2013).

[8] E. L. Rempel, A. C.-L. Chian, and A. Brandenburg, Astrophys. J. Lett. 735, L9 (2011).

[9] E. L. Rempel, T. F. P. Gomes, S. S. A. Silva, and A. C.-L. Chian, Phys. Rev. E 99, 043206 (2019).

[10] E. E. Lawrence and W. Gekelman, Phys. Rev. Lett. 103, 105002 (2009); W. Gekelman, T. DeHaas, W. Daughton, B. Van Compernolle, T. Intrator, and S. Vincena, ibid. 116, 235101 (2016).

[11] N. Wang, S. Datta-Barua, A. T. Chartier, U. Ramirez, and C. N. Mitchell, J. Geophys. Res. 123, 5831 (2018); U. Ramirez, N. Wang, A. T. Chartier, and S. Datta-Barua, ibid. 124, 3573 (2019).

[12] A. R. Yeates, G. Hornig, and B. T. Welsch, Astron. Astrophys. 539, A1 (2012).

[13] A. C.-L. Chian, E. L. Rempel, G. Aulanier, B. Schmieder, S. C. Shadden, B. T. Welsch, and A. R. Yeates, Astrophys. J. 786, 51 (2014).

[14] F. Rincon and M. Rieutord, Liv. Rev. Solar Phys. 15, 6 (2018).

[15] S. S. A. Silva, E. L. Rempel, T. F. P. Gomes, I. S. Requerey, and A. C.-L. Chian, Astrophys. J. Lett. 863, L2 (2018).

[16] S. S. A. Silva, V. Fedun, G. Verth, E. L. Rempel, and S. Shelyag, Astrophys. J. 898, 137 (2020).

[17] Y. Lai and T. Tél, Transient Chaos: Complex Dynamics on Finite-Time Scales (Springer, Berlin, 2011); T. Tél, Chaos 25, 097619 (2015); H. Aref, J. R. Blake, M. Budišić, S. S. S. Cardoso, J. H. E. Cartwright, H. J. H. Clercx, K. El Omari, U.
Feudel, R. Golestanian, E. Gouillart et al., Rev. Mod. Phys. 89, 025007 (2017).

[18] J. D. Skufca, J. A. Yorke, and B. Eckhardt, Phys. Rev. Lett. 96, 174101 (2006); P. R. Muñoz, J. J. Barroso, A. C.-L. Chian, and E. L. Rempel, Chaos 22, 033120 (2012); A. C.-L. Chian, P. R. Muñoz, and E. L. Rempel, Phys. Rev. E 88, 052910 (2013).

[19] E. C. Silva, I. L. Caldas, R. L. Viana, and M. A. F. Sanjuán, Phys. Plasmas 9, 4917 (2002); J. S. E. Portela, I. L. Caldas, R. L. Viana, and M. A. F. Sanjuán, Int. J. Bifurcation Chaos 17, 4067 (2007).

[20] E. L. Rempel and A. C.-L. Chian, Int. J. Bifurcation Chaos 14, 4009 (2004); A. C.-L. Chian, W. M. Santana, E. L. Rempel, F. A. Borotto, T. Hada, and Y. Kamide, Non. Proc. Geophys. 14, 17 (2007).

[21] E. L. Rempel and A. C.-L. Chian, Phys. Rev. Lett. 98, 014101 (2007); A. C.-L. Chian, R. A. Miranda, E. L. Rempel, Y. Saiki, and M. Yamada, ibid. 104, 254102 (2010); A. C. Mathias, R. L. Viana, T. Kroetz, and I. L. Caldas, Physica A 469, 681 (2017).

[22] E. L. Rempel, G. Lesur, and M. R. E. Proctor, Phys. Rev. Lett. 105, 044501 (2010); A. Riols, F. Rincon, C. Cossu, G. Lesur, P.-Y. Longaretti, G. I. Ogilvie, and J. Herault, J. Fluid Mech. 731, 1 (2013).

[23] F. F. Franco, E. L. Rempel, and P. R. Muñoz, Physica D 406, 132417 (2020).

[24] A. de la Cámara, C. R. Mechoso, A. M. Mancho, E. Serrano, and K. Ide, J. Atmos. Sci. 70, 2982 (2013); A. Guha, C. R. Mechoso, C. S. Konor, and R. P. Heikes, ibid. 73, 393 (2016); M. Zsugyel, T. Tél, and J. Józsa, Adv. Water Res. 71, 81 (2014); T. Haszpra, Chaos 29, 071103 (2019).

[25] T. Roudier, B. Schmieder, B. Filippov, R. Chandra, and J. M. Malherbe, Astron. Astrophys. 618, A43 (2018).

[26] D. E. Innes, A. Genetelli, R. Attie, and H. E. Potts, Astron. Astrophys. 495, 319 (2009).

[27] R. Chertovskih, E. V. Chimanski, and E. L. Rempel, Europhys. Lett. 112, 14001 (2015).

[28] D. Orozco Suárez, Y. Katsukawa, and L. R. Bellot Rubio, Astrophys. J. Lett. 758, L38 (2012).

[29] I. S. Requerey, B. Ruiz Cobo, Milan Gošić, and L. R. Bellot Rubio, Astron. Astrophys. 610, A84 (2018). 
[30] M. Verma, M. Steffen, and C. Denker, Astron. Astrophys. 555, A136 (2013).

[31] S. C. Shadden, F. Lekien, and J. E. Marsden, Physica D 212, 271 (2005).

[32] P. Démoulin, E. R. Priest, and D. P. Lonie, J. Geophys. Res. 101, 7631 (1996).

[33] M. Mathur, G. Haller, T. Peacock, J. E. Ruppert-Felsot, and H. L. Swinney, Phys. Rev. Lett. 98, 144502 (2007).

[34] M. Farazmand and G. Haller, Chaos 23, 023101 (2013).

[35] D. Jánosi and T. Tél, Chaos 29, 121105 (2019).

[36] T. Tél, L. Kadi, I. M. Janosi, and M. Vincze, Europhys. Lett. 123, 44001 (2018).
[37] I. N. Kitiashvili, A. G. Kosovichev, N. N. Mansour, S. K. Lele, and A. A. Wray, Phys. Scr. 86, 018403 (2012).

[38] K. Kusano, Y. Bamba, T. T. Yamamoto, Y. Iida, S. Toriumi, and A. Asai, Astrophys. J. 760, 31 (2012); K. Kusano, T. Iju, Y. Bamba, and S. Inoue, Science 369, 587 (2020).

[39] S.-H. Park, K. D. Leka, K. Kusano, J. Andries, G. Barnes, S. Bingham, D. Shaun Bloomfield, A. E. McCloskey, V. Delouille, D. Falconer et al., Astrophys. J. 890, 124 (2020).

[40] Handbook of the Solar-Terrestrial Environment, edited by Y. Kamide and A. C.-L. Chian (Springer, Berlin, 2007).

[41] A. T. Semple, Meteorol. Appl. 10, 39 (2003).

[42] H. E. Huppert and K. Bryan, Deep-Sea Res. 23, 655 (1976). 